\title{
The Gaussian free field and strict plane partitions
}

\author{
Mirjana Vuletić
}

University of Massachusetts Boston, Mathematics Department, Boston, MA 02125, USA

\begin{abstract}
We study height fluctuations around the limit shape of a measure on strict plane partitions. It was shown in our earlier work that this measure is a Pfaffian process. We show that the height fluctuations converge to a pullback of the Green's function for the Laplace operator with Dirichlet boundary conditions on the first quadrant. We use a Pfaffian formula for higher moments to show that the height fluctuations are governed by the Gaussian free field. The results follow from the correlation kernel asymptotics which is obtained by the steepest descent method.
\end{abstract}

Résumé. Nous étudions les fluctuations de la hauteur autour de la forme limite d'une mesure sur les partitions planes strictes. Nous avons déjà montré que cette mesure est un processus Pfaffien. Nous montrons que les fluctuations convergent vers un "pullback" de la fonction de Green pour l'opérateur de Laplace avec des conditions de bord de Dirichlet sur le premier quadrant. Nous utilisons une formule Pfaffienne pour les moments d'ordre supérieur pour montrer que les fluctuations sont gouvernées par le champ libre gaussien. Ces résultats découlent de l'asymptotique du noyau de corrélation qui est obtenue par la méthode du col.

Keywords: Gaussian free field, Pfaffian process, limit shape, height function fluctuations, plane partitions, correlation function asymptotics

\section{Introduction}

In this paper we study fluctuations of the height function around the limit shape of a measure on strict plane partitions that was introduced in our earlier paper [V]. In that earlier paper, we found the correlation function for this measure and showed that it is given in terms of a Pfaffian. We used the correlation function to obtain the limit shape of large strict plane partitions. In this paper, we study fluctuations around the limit shape. We show that the fluctuations are governed by the Gaussian free field. We do this by finding the leading term in the asymptotic expansion of the correlation kernel using the steepest descent method.

Kenyon was the first to show that the Gaussian free field describes height fluctuations of two random surface models, see [K1, K2]. After that there have been other similar results [BF, D, Ku, P, RV]. All these processes are determinantal. Here we present a Pfaffian process whose fluctuations are given by the Gaussian free field.

We start by explaining our results in more detail. 


\subsection{Measure on strict plane partitions}

A plane partition is a filling of a Young diagram with positive integers in such a way that each row and each column is a non-increasing sequence of integers, see the left part of Figure 1 A plane partition $\pi$ can be represented as a 3-dimensional diagram (object), by stacking $\pi_{i, j}$ cubes above the $(i, j)$-box of its Young diagram, see the right part of Figure 1 . The weight of a plane partition $\pi$, denoted with $|\pi|$, is the sum of
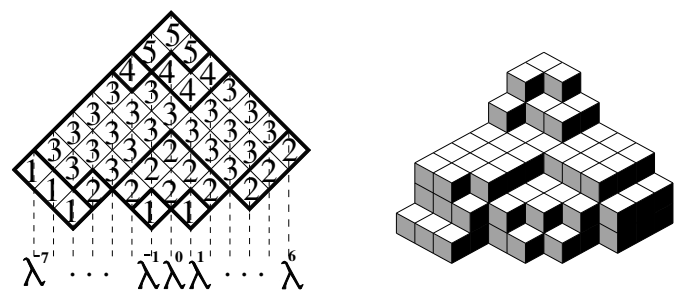

Fig. 1: A plane partition and its 3-dimensional diagram

all entries, or alternatively, the volume of the 3-dimensional diagram. A connected component of a plane partition is a set of rook-wise connected boxes filled with a same integer. In the 3-dimensional diagram connected components are white terraces seen from the above. The number of connected components of a plane partition $\pi$ is denoted with $k(\pi)$. For the plane partition shown in Figure 1 the number of connected components is 10 .

Diagonals of a plane partition are all (ordinary) partitions. A (diagonally) strict plane partition is a partition whose all diagonals are strict partitions. A strict plane partition $\pi$ can be represented with a 2-dimensional diagram, which is a subset of

$$
\mathfrak{X}=\{(t, x) \in \mathbb{Z} \times \mathbb{Z} \mid x>0\}
$$

containing points $\left(j-i, \pi_{i, j}\right)$, where $(i, j)$ is a box in the Young diagram of $\pi$ and $\pi_{i, j}$ is its filling number, see Figure 2
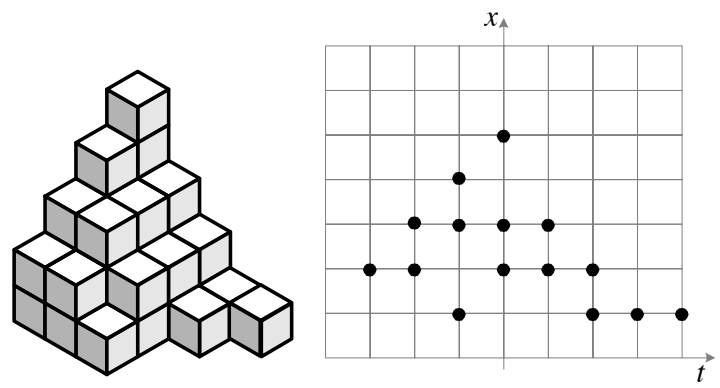

Fig. 2: A strict plane partition and its 2-dimensional diagram

A probability measure $\mathfrak{M}_{q}$ on the set of strict plane partitions is defined by

$$
\mathfrak{M}_{q}(\pi) \propto 2^{k(\pi)} q^{|\pi|} .
$$


The normalization constant is given by the shifted MacMahon formula, see [V]:

$$
\sum_{\substack{\pi \text { is a strict } \\ \text { plane partition }}} 2^{k(\pi)} q^{|\pi|}=\prod_{n=1}^{\infty}\left(\frac{1+q^{n}}{1-q^{n}}\right)^{n} .
$$

The limit shape of large strict plane partitions distributed according to $\mathfrak{M}_{q}$ is shown in Figure 3 , see [V]. It is parameterized on the domain $\mathcal{D}$ representing a half of the amoeba of the polynomial $P(z, w)=$ $-1+z+w+z w$, see (1) for the definition.
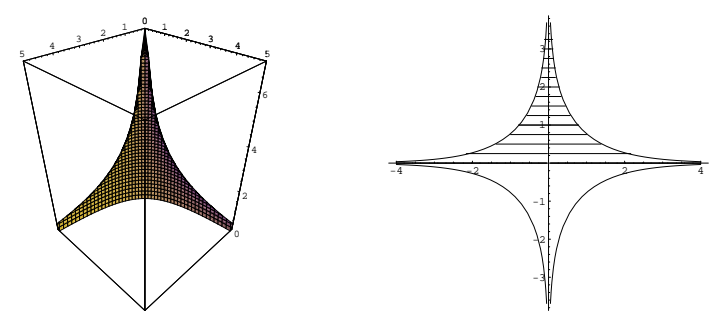

Fig. 3: The limit shape and the half-amoeba (the domain of the limit shape parametrization)

The limit shape for $\tau \geq 0$ (symmetrically for $\tau<0$ ) is given by

$$
\begin{aligned}
& x(\tau, \chi)=R(\tau, \chi), \\
& y(\tau, \chi)=R(\tau, \chi)+\tau, \\
& z(\tau, \chi)=\chi
\end{aligned}
$$

for $(\tau, \chi) \in \mathcal{D}$, where

$$
\mathcal{D}=\left\{(\xi, \omega)=(\log |z|, \log |w|) \in \mathbb{R}^{2}, \omega \geq 0 \mid(z, w) \in(\mathbb{C} \backslash\{0\})^{2},-1+z+w+z w=0\right\} .
$$

and $R(\tau, \chi)$ is the Ronkin function of $-1+z+w+z w$ at $(\tau / 2,-\chi / 2)$, i.e.

$$
R(\tau, \chi)=\frac{1}{2 \pi^{2}} \int_{0}^{2 \pi} \int_{0}^{2 \pi} \log \left|1-e^{\tau / 2+i u}-e^{-\chi / 2+i v}-e^{\tau / 2+i u-\chi+i v}\right| d u d v .
$$

The limit shape was obtained using the correlation function that can be written as a Pfaffian of a certain skew-symmetric matrix, more precisely, we exploit the fact that the process is Pfaffian, see below for a definition.

\subsection{Determinantal and Pfaffian processes}

Let $\mathcal{X}$ be a discrete space. A random point process $P$ on $\mathcal{X}$ is a probability measure on the set $2^{\mathcal{X}}$ of all subsets of $\mathcal{X}$. For $x_{1}, x_{2}, \ldots, x_{n} \in \mathcal{X}$, the correlation function $\rho$ is

$$
\rho\left(x_{1}, x_{2}, \ldots, x_{n}\right)=\operatorname{Pr}\left(X \in 2^{\mathcal{X}} \mid\left\{x_{1}, x_{2}, \ldots, x_{n}\right\} \subset X\right) .
$$


$P$ is called a determinantal process if there exists a function $K(x, y)$ such that for any finite set $\left\{x_{1}, x_{2}, \ldots, x_{n}\right\} \subset \mathcal{X}$ one has

$$
\rho\left(x_{1}, x_{2}, \ldots, x_{n}\right)=\operatorname{det}\left(K\left(x_{i}, x_{j}\right)\right)_{i, j=1}^{n} .
$$

$K$ is called the correlation kernel of the determinantal point process $P$.

$P$ is called a Pfaffian process if there exists a $2 \times 2$ matrix valued kernel such that $K(x, y)=-K^{T}(y, x)$ and such that for any finite set $\left(x_{1}, x_{2}, \ldots, x_{n}\right) \subset \mathcal{X}$ one has

$$
\rho\left(x_{1}, x_{2}, \ldots, x_{n}\right)=\operatorname{Pf}\left(K\left(x_{i}, x_{j}\right)\right)_{i, j=1}^{n} .
$$

The explicit formula for the correlation kernel of $\mathfrak{M}_{q}$ is given in Section 2 and was derived in our earlier paper. In that paper, we started asymptotic analysis of the correlation kernel and computed the limit shape as a result. Here, we continue with the asymptotic analysis and obtain that the height fluctuations around the limit shape are given by a pullback of the Gaussian free field on the first quadrant. We define the Gaussian free field below.

\subsection{The Gaussian free field}

Continuous Gaussian free field is a random field (collection of random variables) over a domain $D \in \mathbb{R}^{d}$. The collection consists of zero mean Gaussians with the covariance

$$
E\left[G F F\left(z_{1}\right) G F F\left(z_{2}\right)\right]=G\left(z_{1}, z_{2}\right),
$$

where $G\left(z_{1}, z_{2}\right)$ is the Green's function for the Laplacian on $D$ (i.e. $\left.\Delta G\left(z_{1}, z_{2}\right)=\delta_{z_{1}}\left(z_{2}\right)\right)$ satisfying the Dirichlet boundary conditions (i.e. $G$ vanishes on $\partial D$ ).

In the case $d=1$ the Gaussian free field is either the Brownian motion or Brownian bridge. In higher dimensions we need to define it as a random generalized function. Let $C_{0}^{1}(D)$ be the set of test functions on $D$, i.e. smooth compactly supported functions on $D$, and let $H(D)$ be its Hilbert space closure under the Dirichlet inner product

$$
\left\langle\varphi_{1}, \varphi_{2}\right\rangle=\int_{D} \nabla \varphi_{1}(z) \cdot \nabla \varphi_{2}(z)|d z|^{2}=\left\langle\varphi_{1}, \varphi_{2}\right\rangle=\int_{D} \int_{D} \varphi_{1}\left(z_{1}\right) \varphi_{2}\left(z_{2}\right) G\left(z_{1}, z_{2}\right)\left|d z_{1}\right|^{2}\left|d z_{2}\right|^{2} .
$$

The Gaussian free field $G F F$ is the formal sum $\sum_{i} \alpha_{i} f_{i}$, where $\left\{f_{i}\right\}$ is an orthonormal basis for $H(D)$ and $\alpha_{i}$ are i.i.d. standard Gaussians.

The higher moments of $G F F$ (in the generalized function sense) are

$$
E\left[G F F\left(z_{1}\right) \cdots G F F\left(z_{n}\right)\right]=\left\{\begin{array}{ll}
\sum_{\sigma} \prod_{i=1}^{n / 2} G\left(z_{\sigma(2 i-1)}, z_{\sigma(2 i)}\right) & n \text { is even } \\
0 & n \text { is odd }
\end{array},\right.
$$

where the sum is taken over all fixed point free involutions $\sigma$ on $\{1,2, \ldots, n\}$.

Any process whose higher moments are given by the formula above is the Gaussian free field. In this paper we use this property to show that height fluctuations are governed by the Gaussian free field on the first quadrant $Q \subset \mathbb{R}^{2}$. The Green's function for the Laplace operator with the Dirichlet boundary conditions on $Q$ is given by

$$
G\left(z_{1}, z_{2}\right)=\frac{1}{2 \pi} \log \left|\frac{\left|z_{1}-z_{2}\right|\left|z_{1}+z_{2}\right|}{\left|\bar{z}_{1}-z_{2}\right|\left|\bar{z}_{1}+z_{2}\right|}\right|
$$




\subsection{Results}

The Gaussian free field is a random object that has been associated with many determinantal processes: uniform planar domino tilings [K1], a honeycomb dimer model [K2], a KPZ class model [BF], interlacing particles $[\overline{\mathrm{D}}, \mathrm{Ku}]$, uniformly random lozenge tilings of polygons $[\overline{\mathrm{P}}]$, and a random matrix model [RV]. For all these models it has been shown that certain fluctuations behave like the Gaussian free field.

In this paper, we show that this holds for our model too. As far as we know, this is the first time where the Gaussian free field is associated with a Pfaffian process.

For a plane partition $\pi$ and $(t, x) \in \mathfrak{X}$, we define the height function $h_{\pi}(t, x)$ by

$$
h_{\pi}(t, x)=\#\{(t, y) \in \pi \mid y \geq x\} .
$$

In the 3-dimensional diagram of $\pi$, the height function represents the distance between the top surface and the right wall for $t \geq 0$ and the left wall for $t<0$, see Figure 2

For $(t, x) \in \mathfrak{X}$, we define the height fluctuation by

$$
H(t, x)=\sqrt{\pi}[h(t, x)-E(h(t, x))] .
$$

The main result of this paper is that there is a map between the domain $\mathfrak{X}$, representing strict plane partitions, and the quadrant $Q$ under which the pushforward of $H$ converges to the Gaussian free field on $Q$.

In this paper we only give an outline of the proofs. The full version of the paper with details will be published somewhere else. The paper is organized as follows. In Section 2 we recall the correlation function of $\mathfrak{M}_{q}$ which was derived in [V]. In Section 3 we derive the main terms in the asymptotic expansion of the correlation kernel. In Section 4 we use the asymptotic formula to obtain that the covariance of the height fluctuation is given by the Green's function (2). In Section 5 we show that the fluctuations are given by the pullback of the Gaussian free field on the first quadrant. We finish with some possible directions in which this work can be continued. This is given in Section 6 .

\section{Background}

The correlation kernel for the measure $\mathfrak{M}_{q}$ was derived in [V].

Let

$$
J_{q}(t, z)= \begin{cases}\frac{\left(q^{1 / 2} z^{-1} ; q\right)_{\infty}\left(-q^{t+1 / 2} z ; q\right)_{\infty}}{\left(-q^{1 / 2} z^{-1} ; q\right)_{\infty}\left(q^{t+1 / 2} z ; q\right)_{\infty}} & t \geq 0, \\ \frac{\left(-q^{1 / 2} z ; q\right)_{\infty}\left(q^{-t+1 / 2} z^{-1} ; q\right)_{\infty}}{\left(q^{1 / 2} z ; q\right)_{\infty}\left(-q^{-t+1 / 2} z^{-1} ; q\right)_{\infty}} & t<0\end{cases}
$$

where

$$
(z ; q)_{\infty}=\prod_{n=0}^{\infty}\left(1-q^{n} z\right)
$$

is the quantum dilogarithm function. 
Theorem 2.1 $[\nabla]$ Let $X=\left\{\left(t_{i}, x_{i}\right): i=1, \ldots, n\right\} \subset \mathfrak{X}$. The correlation function has the form

$$
\rho(X)=\operatorname{Pf}\left(M_{X}\right)
$$

where $M_{X}$ is a skew-symmetric $2 n \times 2 n$ matrix

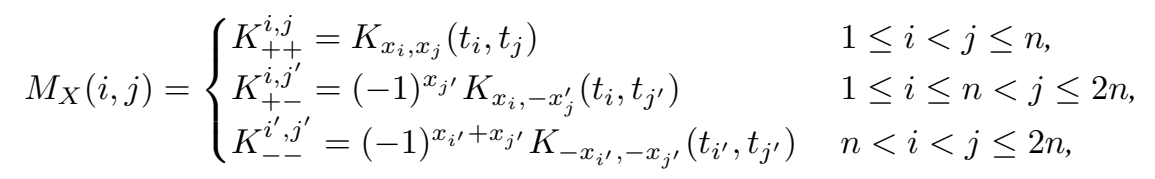

where $i^{\prime}=2 n-i+1$ and $K_{x, y}\left(t_{i}, t_{j}\right)$ is the coefficient of $z^{x} w^{y}$ in the formal power series expansion of

$$
\frac{z-w}{2(z+w)} J_{q}\left(z, t_{i}\right) J_{q}\left(w, t_{j}\right)
$$

in the region $|z|>|w|$ if $t_{i} \geq t_{j}$ and $|z|<|w|$ if $t_{i}<t_{j}$.

By the definition,

$$
\begin{aligned}
K_{++}^{i, j} & =\frac{1}{(2 \pi i)^{2}} \iint_{\substack{|z|=1 \pm \epsilon \\
|w|=1 \mp \epsilon}} \frac{z-w}{2(z+w)} J_{q}\left(t_{i}, z\right) J_{q}\left(t_{j}, w\right) \frac{1}{z^{x_{i}+1} w^{x_{j}+1}} d z d w \\
K_{+-}^{i, j} & =\frac{(-1)^{x_{j}}}{(2 \pi i)^{2}} \iint_{\substack{|z|=1 \pm \epsilon \\
|w|=1 \mp \epsilon}} \frac{z-w}{2(z+w)} J_{q}\left(t_{i}, z\right) J_{q}\left(t_{j}, w\right) \frac{1}{z^{x_{i}+1} w^{-x_{j}+1}} d z d w \\
K_{--}^{i, j} & =\frac{(-1)^{x_{i}+x_{j}}}{(2 \pi i)^{2}} \iint_{\substack{|z|=1 \pm \epsilon \\
|w|=1 \mp \epsilon}} \frac{z-w}{2(z+w)} J_{q}\left(t_{i}, z\right) J_{q}\left(t_{j}, w\right) \frac{1}{z^{-x_{i}+1} w^{-x_{j}+1}} d z d w
\end{aligned}
$$

where we take the upper signs if $t_{i} \geq t_{j}$ and the lower signs otherwise.

Observe that $J_{q}(-z)=1 / J_{q}(z)$. If we make the change of variables $w \mapsto-w$ in the second kernel and $z \mapsto-z$ and $w \mapsto-w$ in the third one we obtain

$$
\begin{aligned}
K_{++}^{i, j} & =\frac{1}{(2 \pi i)^{2}} \iint_{\substack{|z|=1 \pm \epsilon \\
|w|=1 \mp \epsilon}} \frac{z-w}{2(z+w)} J_{q}\left(t_{i}, z\right) J_{q}\left(t_{j}, w\right) \frac{1}{z^{x_{i}+1} w^{x_{j}+1}} d z d w \\
K_{+-}^{i, j} & =\frac{1}{(2 \pi i)^{2}} \iint_{\substack{|z|=1 \pm \epsilon \\
|w|=1 \mp \epsilon}} \frac{z+w}{2(z-w)} \frac{J_{q}\left(t_{i}, z\right)}{J_{q}\left(t_{j}, w\right)} \frac{1}{z^{x_{i}+1} w^{-x_{j}+1}} d z d w \\
K_{--}^{i, j} & =\frac{1}{(2 \pi i)^{2}} \iint_{\substack{|z|=1 \pm \epsilon \\
|w|=1 \mp \epsilon}} \frac{z-w}{2(z+w)} \frac{1}{J_{q}\left(t_{i}, z\right) J_{q}\left(t_{j}, w\right)} \frac{1}{z^{-x_{i}+1} w^{-x_{j}+1}} d z d w
\end{aligned}
$$




\section{Asymptotics of the correlation kernel}

Let $\left(\tau_{i}, \chi_{i}\right)$ and $\left(\tau_{j}, \chi_{j}\right)$ be two different fixed points in $\mathcal{D}$ and $r=-\log q$. We are interested in finding the first terms in the asymptotic expansion of the kernels $K_{++}^{i, j}, K_{+-}^{i, j}$ and $K_{--}^{i, j}$ where $r t_{i} \rightarrow \tau_{i}, r t_{j} \rightarrow \tau_{j}$, $r x_{i} \rightarrow \chi_{i}$ and $r x_{j} \rightarrow \chi_{j}$ when $r \rightarrow+0$. We do this using the steepest descent method.

We start with a simpler problem first. Let $r t \rightarrow \tau$ and $r x \rightarrow \chi$ when $r \rightarrow+0$ for $(\tau, \chi) \in \mathcal{D}$. We want to find the main term in the asymptotic expansion of

$$
I=\frac{1}{2 \pi i} \int_{|z|=e^{\tau / 2}} \frac{1}{z} J_{q}(t, z)\left(\frac{z}{e^{\tau / 2}}\right)^{-x} d z .
$$

This integral contains terms of the form

$$
\exp \left[\log (z ; q)_{\infty}\right]
$$

see 33, so we need the asymptotic behavior of $\log (z ; q)_{\infty}$. We have that

$$
\log (z ; q)_{\infty}=-\frac{1}{r} \operatorname{dilog}(z)-\frac{1}{2} \log (1-z)+O(r), \quad r \rightarrow+0,
$$

where

$$
\operatorname{dilog}(z)=\sum_{k=1}^{\infty} \frac{z^{k}}{k^{2}}
$$

is the dilogarithm function which is an analytic function everywhere in $\mathbb{C}^{2} \backslash(1,+\infty)$.

Then the exponentially large term in (4) that determines the asymptotics is

$$
\exp \left[\log \left[J_{q}(t, z)\left(z / e^{\tau / 2}\right)^{-x}\right]\right]=\exp \left[\frac{1}{r} S(z, \tau, \chi)\right][G(z, \tau)+O(r)], \quad r \rightarrow+0,
$$

where

$$
S(z, \tau, \chi)=-\operatorname{dilog}\left(-e^{-\tau} z\right)-\operatorname{dilog}\left(z^{-1}\right)+\operatorname{dilog}\left(e^{-\tau} z\right)+\operatorname{dilog}\left(-z^{-1}\right)-\chi(\log z-\tau / 2)
$$

and

$$
G(z, \tau)=\sqrt{\frac{\left(1+e^{-\tau} z\right)(z-1)}{\left(1-e^{-\tau} z\right)(z+1)}},
$$

with branch cuts $\left(-\infty,-e^{\tau}\right),(-1,0),(0,1)$ and $\left(e^{\tau}, \infty\right)$.

So, we need to find the main term in the asymptotic expansion of

$$
\frac{1}{2 \pi i} \int_{|z|=e^{\tau / 2}} \frac{1}{z} G(z, \tau) \exp \left[\frac{1}{r} S(z, \tau, \chi)\right] d z .
$$

We deform the contour $|z|=e^{\tau / 2}$ to a new contour $\gamma$ such that $\operatorname{Re} S(z, \tau, \chi)<0$ except at the two critical points that lie on $|z|=e^{\tau / 2}$. In addition, we choose $\gamma$ that passes through two line segments in 
the direction of the steepest descent of $\operatorname{Re} S$, which we denote with $\gamma_{\text {loc }}$ and $-\bar{\gamma}_{\text {loc }}$. The main asymptotic term will come from the portion of the integral obtained by the integration over these line segments.

The two critical points are $z_{c}=z(\tau, \chi)$ and $\bar{z}_{c}=\overline{z(\tau, \chi)}$, where

$$
z(\tau, \chi)=\exp \left[\tau / 2+i \arccos \frac{\left(e^{\tau}+1\right)\left(e^{\chi}-1\right)}{2 e^{\tau / 2}\left(e^{\chi}+1\right)}\right] .
$$

The two line segments in the direction of the steepest descent of $\operatorname{Re} S, \gamma_{\text {loc }}$ and $-\bar{\gamma}_{\text {loc }}$, are given by

$$
\gamma_{\mathrm{loc}}=z_{\mathrm{c}}+\hat{\theta}_{z_{\mathrm{c}}} x, \quad \hat{\theta}_{z_{\mathrm{c}}}=e^{i \gamma_{z_{\mathrm{c}}}}, x \in[-\delta, \delta], \delta>0
$$

where

$$
\gamma_{z_{c}}=\frac{\pi}{2}-\frac{\arg S^{\prime \prime}\left(z_{c}, \tau, \chi\right)}{2}=\frac{\pi}{4}+\arg \left(z_{c}\right) \text {. }
$$

Let $\gamma_{1}$ be the portion of $\gamma$ without $\gamma_{\text {loc }}$ and $-\bar{\gamma}_{\text {loc }}$. Let $M>0$ be such that $\operatorname{Re}(S(z, \tau, \chi)) \leq-M$ on $\gamma_{1}$. Then the portion of the integral coming from the integration over $\gamma_{1}$ is

$$
I_{1}=\frac{1}{2 \pi i} \int_{\gamma_{1}} \frac{1}{z} G(z, \tau) \exp \left[\frac{1}{r} S(z, \tau, \chi)\right]=O(\exp [-M / r])
$$

Now, for

$$
\begin{aligned}
I_{\mathrm{loc}} & =\frac{1}{2 \pi i} \int_{\gamma_{\mathrm{loc}}} \frac{1}{z} G(z, \tau) \exp \left[\frac{1}{r} S(z, \tau, \chi)\right] \\
& =\frac{1}{2 \pi i} \int_{-\delta}^{\delta} \frac{1}{z_{\mathrm{c}}}\left(G\left(z_{c}, \tau\right)+O(x)\right) \exp \left[\frac{1}{r} S\left(z_{c}, \tau, \chi\right)-\frac{1}{2 r} x^{2}\left|S^{\prime \prime}\left(z_{c}, \tau, \chi\right)\right|+\frac{1}{r} O\left(x^{3}\right)\right] \hat{\theta}_{z_{\mathrm{c}}} d x
\end{aligned}
$$

we get (using Gaussian integral)

$$
I_{\mathrm{loc}}=\frac{1}{2 \pi i} \exp \left[\frac{1}{r} S\left(z_{c}, \tau, \chi\right)\right] \frac{\hat{\theta}_{z_{\mathrm{c}}}}{z_{\mathrm{c}}} G\left(z_{c}, \tau\right) \sqrt{\frac{2 \pi r}{\left|S^{\prime \prime}\left(z_{c}, \tau, \chi\right)\right|}}(1+O(\sqrt{r}))
$$

Similarly, the integral over $-\bar{\gamma}_{\text {loc }}$ is

$$
\frac{1}{2 \pi i} \exp \left[\frac{1}{r} S\left(\bar{z}_{c}, \tau, \chi\right)\right] \frac{\hat{\theta}_{\bar{z}_{\mathrm{c}}}}{\bar{z}_{\mathrm{c}}} G\left(\bar{z}_{c}, \tau\right) \sqrt{\frac{2 \pi r}{\left|S^{\prime \prime}\left(\bar{z}_{c}, \tau, \chi\right)\right|}}(1+O(\sqrt{r})) .
$$

Finally, combining

$$
I=\operatorname{Re}\left(\frac{1}{\pi i} \exp \left[\frac{1}{r} \operatorname{Im} S\left(z_{c}, \tau, \chi\right)\right] \frac{\hat{\theta}_{z_{\mathrm{c}}}}{z_{\mathrm{c}}} G\left(z_{c}, \tau\right) \sqrt{\frac{2 \pi r}{\left|S^{\prime \prime}\left(z_{c}, \tau, \chi\right)\right|}}\right)(1+O(\sqrt{r}))
$$




\subsection{Correlation kernel asymptotics}

Now, we are ready to find the main terms in the asymptotic expansions of the kernels $K_{++}^{i, j}, K_{+-}^{i, j}$ and $K_{--}^{i, j}$. For convenience, we take out exponential terms in the expressions for the kernels (they cancel out in the Pfaffian formula for the correlation function). For example,

$$
K_{++}^{i, j}=e^{\left(-\tau_{i} x_{i}-\tau_{j} x_{j}\right) / 2} \cdot I_{++}^{i, j},
$$

where

$$
I_{++}^{i, j}=\frac{1}{(2 \pi i)^{2}} \iint \frac{z-w}{2 z w(z+w)} J_{q}\left(t_{i}, z\right) J_{q}\left(t_{j}, w\right)\left(\frac{z}{e^{\tau_{i} / 2}}\right)^{-x_{i}}\left(\frac{w}{e^{\tau_{j} / 2}}\right)^{-x_{j}} d z d w,
$$

with integration contours $|z|=e^{\tau_{i} / 2}$ and $|w|=e^{\tau_{j} / 2}$.

The main term in the asymptotics of $I_{++}^{i, j}$ will be the same as the main term in the asymptotics of

$$
\frac{1}{(2 \pi i)^{2}} \iint \frac{z-w}{2 z w(z+w)} G\left(z, \tau_{i}\right) G\left(w, \tau_{j}\right) \exp \left[\frac{1}{r}\left[S\left(z, \tau_{i}, \chi_{i}\right)+S\left(w, \tau_{j}, \chi_{j}\right)\right]\right] d z d w,
$$

with integration contours $|z|=e^{\tau_{i} / 2}$ and $|w|=e^{\tau_{j} / 2}$.

Using the same steepest descent analysis as for $I$ we get the following:

Theorem 3.1 When $r \rightarrow 0+$

$$
\begin{aligned}
I_{++}^{i j} & \approx \frac{r}{4 \pi} \bar{\sum} \frac{z_{c}-w_{c}}{z_{c}+w_{c}} \frac{1}{z_{c} w_{c}} \frac{1}{\sqrt{S^{\prime \prime}\left(z_{c}\right) S^{\prime \prime}\left(w_{c}\right)}} F\left(z_{c}\right) F\left(w_{c}\right) \\
I_{+-}^{i j} & \approx \frac{r i}{4 \pi} \bar{\sum} \frac{z_{c}+w_{c}}{z_{c}-w_{c}} \frac{1}{z_{c} w_{c}} \frac{1}{\sqrt{S^{\prime \prime}\left(z_{c}\right) S^{\prime \prime}\left(w_{c}\right)}} \frac{F\left(z_{c}\right)}{F\left(w_{c}\right)}(-1)^{\mathbb{1}\left(w_{c}\right)} \\
I_{--}^{i j} & \approx \frac{r}{4 \pi} \bar{\sum} \frac{z_{c}-w_{c}}{z_{c}+w_{c}} \frac{1}{z_{c} w_{c}} \frac{1}{\sqrt{S^{\prime \prime}\left(z_{c}\right) S^{\prime \prime}\left(w_{c}\right)}} \frac{1}{F\left(z_{c}\right) F\left(w_{c}\right)}(-1)^{\mathbb{1}\left(z_{c}\right)+\mathbb{1}\left(w_{c}\right)},
\end{aligned}
$$

where

$$
\begin{gathered}
\bar{\sum} f(z, w)=f(z, w)+f(\bar{z}, w)+f(z, \bar{w})+f(\bar{z}, \bar{w}) \\
F(z)=G(z, \tau) \exp \left[\frac{1}{r} S(z, \tau, \chi)\right], \quad \mathbb{1}(z)= \begin{cases}0 & \operatorname{Re} z>0 \\
1 & \operatorname{Re} z<0\end{cases} \\
z_{c}=z\left(\tau_{i}, \chi_{i}\right), w_{c}=z\left(\tau_{j}, \chi_{j}\right) .
\end{gathered}
$$

\section{Covariance}

Recall, the height function $h_{\pi}(t, x)$ is defined by $h_{\pi}(t, x)=\#\{(t, y) \in \pi \mid y \geq x\}$ and fluctuations by $H(t, x)=\sqrt{\pi}[h(t, x)-E(h(t, x))]$. We want to show

\section{Theorem 4.1}

$$
\lim _{r \rightarrow 0+} E\left(H\left(t_{1}, x_{1}\right) H\left(t_{2}, x_{2}\right)\right)=G\left(z_{1}, z_{2}\right)
$$

when $r t_{i} \rightarrow \tau_{i}$ and $r x_{i} \rightarrow \chi_{i}$ for $\left(\tau_{i}, \chi_{i}\right) \in \mathcal{D}$, where $z_{i}=z\left(\tau_{i}, \chi_{i}\right)$, see (5). G is the Green's function given by (2). 
We start with

$$
\begin{aligned}
& E\left(h\left(t_{1}, x_{1}\right) h\left(t_{2}, x_{2}\right)\right)-E\left(h\left(t_{1}, x_{1}\right)\right) E\left(h\left(t_{2}, x_{2}\right)\right) \\
& =\sum_{y_{1} \geq x_{1}} \sum_{y_{2} \geq x_{2}} \rho_{2}\left(\left(t_{1}, y_{1}\right),\left(t_{2}, y_{2}\right)\right)-\sum_{y_{1} \geq x_{1}} \rho_{1}\left(t_{1}, y_{1}\right) \sum_{y_{2} \geq x_{2}} \rho_{1}\left(t_{2}, y_{2}\right) \\
& =\sum_{y_{1} \geq x_{1}} \sum_{y_{2} \geq x_{2}}\left(\operatorname{Pf}\left[\begin{array}{cccc}
0 & K_{++}^{y_{1}, y_{2}} & K_{+-}^{y_{1}, y_{2}} & K_{+}^{y_{1}, y_{1}} \\
& 0 & K_{+-}^{y_{2}, y_{2}} & K_{+}^{y_{2}, y_{1}} \\
& & 0 & K_{--}^{y_{2}, y_{1}} \\
& & & 0
\end{array}\right]-\operatorname{Pf}\left[\begin{array}{cc}
0 & K_{+-}^{y_{1}, y_{1}} \\
& 0
\end{array}\right] \operatorname{Pf}\left[\begin{array}{cc}
0 & K_{+-}^{y_{2}, y_{2}} \\
&
\end{array}\right]\right) \\
& =\sum_{y_{1} \geq x_{1}} \sum_{y_{2} \geq x_{2}} K_{++}^{y_{1}, y_{2}} K_{--}^{y_{2}, y_{1}}-K_{+-}^{y_{1}, y_{2}} K_{+-}^{y_{2}, y_{1}}
\end{aligned}
$$

Since, $K_{++}^{1,2} K_{--}^{2,1}-K_{+-}^{1,2} K_{+-}^{2,1}=I_{++}^{1,2} I_{--}^{2,1}-I_{+-}^{1,2} I_{+-}^{2,1}$ we start with

$$
\lim _{r \rightarrow+0} \sum_{\substack{r y_{1} \geq \chi_{1} \\ y_{1} \in \mathbb{N}}} \sum_{\substack{r \\ y_{2} \geq \chi_{2} \\ y_{2} \in \mathbb{N}}} I_{++}\left(\left(t_{1}, y_{1}\right),\left(t_{2}, y_{2}\right)\right) I_{--}\left(\left(t_{1}, y_{1}\right),\left(t_{2}, y_{2}\right)\right)
$$

By Theorem 3.1 we have that this is equal to

$$
\begin{aligned}
& \lim _{r \rightarrow+0} \sum_{\substack{r y_{1} \geq \chi_{1} \\
y_{1} \in \mathbb{N}}} \sum_{\substack{r y_{2} \geq \chi_{2} \\
y_{2} \in \mathbb{N}}} \frac{r}{4 \pi} \bar{\sum} \frac{z_{c}-w_{c}}{z_{c}+w_{c}} \frac{1}{z_{c} w_{c}} \frac{1}{\sqrt{S^{\prime \prime}\left(z_{c}\right) S^{\prime \prime}\left(w_{c}\right)}} F\left(z_{c}\right) F\left(w_{c}\right) \\
& \cdot \frac{r}{4 \pi} \sum \frac{z_{c}-w_{c}}{z_{c}+w_{c}} \frac{1}{z_{c} w_{c}} \frac{1}{\sqrt{S^{\prime \prime}\left(z_{c}\right) S^{\prime \prime}\left(w_{c}\right)}} \frac{1}{F\left(z_{c}\right) F\left(w_{c}\right)}(-1)^{\mathbb{1}\left(z_{c}\right) \mathbb{1}\left(w_{c}\right),}
\end{aligned}
$$

where $z_{c}=z\left(\tau_{1}, \chi_{1}\right)$ and $w_{c}=z\left(\tau_{2}, \chi_{2}\right)$, see $(5)$. When we expand the product, there are 16 terms in the above expression and in the limit only 4 terms survive. We get that the above limit is equal to

$$
\lim _{r \rightarrow+0} \sum_{\substack{r y_{1} \geq \chi_{1} \\ y_{1} \in \mathbb{N}}} \sum_{\substack{r y_{2} \geq \chi_{2} \\ y_{2} \in \mathbb{N}}} \frac{r^{2}}{(4 \pi)^{2}} \bar{\sum}\left(\frac{z_{c}-w_{c}}{z_{c}+w_{c}}\right)^{2} \frac{1}{z_{c}^{2} w_{c}^{2}} \frac{1}{S^{\prime \prime}\left(z_{c}\right) S^{\prime \prime}\left(w_{c}\right)}(-1)^{\mathbb{1}\left(z_{c}\right) \mathbb{1}\left(w_{c}\right),}
$$

The above sum can be written as an integral

$$
\int_{\chi_{1}}^{b\left(\chi_{1}\right)} \int_{\chi_{2}}^{b\left(\chi_{2}\right)} \frac{1}{4 \pi^{2}} \bar{\sum}\left(\frac{z_{c}-w_{c}}{z_{c}+w_{c}}\right)^{2} \frac{1}{\left(z_{c} w_{c}\right)^{2}} \frac{1}{S^{\prime \prime}\left(z_{c}\right) S^{\prime \prime}\left(w_{c}\right)}(-1)^{\mathbb{1}\left(z_{c}\right) \mathbb{1}\left(w_{c}\right)} d \chi_{1} d \chi_{2},
$$

where $b$ gives the boundary of $\mathcal{D}$. Because $S^{\prime \prime}(z)=\frac{1}{z} \frac{\partial z}{\partial \chi}$, this integral is equal to

$$
\begin{aligned}
& \frac{1}{4 \pi^{2}} \int_{\substack{|z|=e^{\tau / 2} \\
z_{c} \rightarrow \bar{z}_{c}}} \int_{\substack{|w|=e^{\tau / 2} \\
w_{c} \rightarrow \bar{w}_{c}}} \frac{1}{z w}\left(\frac{z-w}{z+w}\right)^{2} d z d w \\
= & \frac{1}{4 \pi^{2}}\left[\log \frac{z_{c}}{\bar{z}_{c}} \log \frac{w_{c}}{\bar{w}_{c}}+2 \log \left|\frac{z_{c}+w_{c}}{\bar{z}_{c}+w_{c}}\right|\right] .
\end{aligned}
$$


Similarly,

$$
\begin{aligned}
& \lim _{r \rightarrow+0} \sum_{\substack{r y_{1} \geq \chi_{1} \\
y_{1} \in \mathbb{N}}} \sum_{\substack{r y_{2} \geq \chi_{2} \\
y_{2} \in \mathbb{N}}} I_{+-}\left(\left(t_{1}, y_{1}\right),\left(t_{2}, y_{2}\right)\right) I_{+-}\left(\left(t_{1}, y_{1}\right),\left(t_{2}, y_{2}\right)\right) \\
= & \frac{1}{4 \pi^{2}}\left[\log \frac{z_{c}}{\bar{z}_{c}} \log \frac{w_{c}}{\bar{w}_{c}}-2 \log \left|\frac{z_{c}-w_{c}}{\bar{z}_{c}-w_{c}}\right|\right] .
\end{aligned}
$$

When we combine both limits (6) and (7) we get

$$
\frac{1}{2 \pi^{2}} \log \frac{\left|z_{c}+w_{c}\right|\left|z_{c}-w_{c}\right|}{\left|\bar{z}_{c}+w_{c}\right|\left|\bar{z}_{c}-w_{c}\right|}=\frac{1}{\pi} G\left(z_{c}, w_{c}\right) \text {. }
$$

\section{Higher moments. The Gaussian free field}

We now show that the height fluctuations are given by the pullback of the Gaussian free field on the first quadrant $Q$ by $z=z(\tau, \chi)$ (defined by 5 )):

Theorem 5.1 Let $r t_{i} \rightarrow \chi_{i}$ and $r x_{i} \rightarrow \tau_{i}$ when $r \rightarrow 0+$ then

$$
\lim _{r \rightarrow 0+} E\left[H\left(t_{1}, x_{1}\right) \cdots H\left(t_{n}, x_{n}\right)\right]=\left\{\begin{array}{ll}
\sum_{\sigma} \prod_{i=1}^{n / 2} G\left(z_{\sigma(2 i-1)}, z_{\sigma(2 i)}\right) & n \text { is even } \\
0 & n \text { is odd }
\end{array},\right.
$$

where the sum is taken over all fixed point free involutions $\sigma$ on $\{1,2, \ldots, n\}$ and $G$ is given by (2).

In order to prove the theorem we first derive a Pfaffian formula for the higher moments.

Lemma 5.2 Let $X=\left\{\left(t_{i}, x_{i}\right): i=1, \ldots, n\right\} \subset \mathfrak{X}$.

$$
E\left[H\left(t_{1}, x_{1}\right) \cdots H\left(t_{n}, x_{n}\right)\right]=(\sqrt{\pi})^{n} \operatorname{Pf}\left[M_{X}^{0}\right]
$$

where $M_{X}^{0}$ is the same as $M_{X}$ in Theorem 2.1 except it has zeros on the minor diagonal too (they both have zeros on the main diagonal).

The formula is derived in a similar way as in the case $n=2$ which was done in Section 4 .

It remains to show that higher moments are given by the sum over fixed point free involutions of the products of the Green's function. This is done using the following lemma.

Lemma 5.3 Let $M=\left(K_{i, j}\right)_{i, j=1 \ldots n}$

$$
K_{i, j}=\left(\begin{array}{cc}
\frac{z_{i}-z_{j}}{z_{i}+z_{j}} & \frac{z_{i}+z_{j}}{z_{i}-z_{j}} \\
\frac{z_{i}+z_{j}}{z_{i}-z_{j}} & \frac{z_{i}-z_{j}}{z_{i}+z_{j}}
\end{array}\right)
$$

Then

$$
\operatorname{Pf}[M]=\left\{\begin{array}{ll}
\sum_{\sigma} \prod_{i=1}^{n / 2} G_{\sigma(2 i-1), \sigma(2 i)} & n \text { is even } \\
0 & n \text { is odd }
\end{array},\right.
$$

where $G_{i, j}=-\frac{8 z_{i} z_{j}\left(z_{i}^{2}+z_{j}^{2}\right)}{\left(z_{i}^{2}-z_{j}^{2}\right)^{2}}$. 


\section{Concluding Remarks}

We have found that the Gaussian free field arises from $\mathfrak{M}_{q}$ which is a Pfaffian process. Previously, this was found only for determinantal processes. We could generalize this result to a class of Pfaffian processes whose kernel possesses certain properties. The properties arise from technical requirements. It would be interesting to construct physical models with these kernels.

We would like to draw attention to Lemma 5.3. As was said before, several determinantal processes were studied that gave rise to the Gaussian free field. The proofs require a small lemma similar to Lemma 5.3 It is interesting that although the models are very different, and that kernels have different asymptotics they all need the same small lemma to transform the determinantal formula for higher moments into the sum of products over different fixed point free involutions. We plan to study other Pfaffian processes in the future and see if they give rise to the Gaussian free field. It would be interesting to see if we would need Lemma 5.3 for the proof. If yes, can we understand why this is the case?

\section{Acknowledgements}

I want to thank Alexei Borodin for suggesting this problem and for many useful discussions.

\section{References}

[BF] A. Borodin and P. L. Ferrari, Anisotropic KPZ growth in 2+1 dimensions: fluctuations and covariance structure; Journal of Statistical Mechanics: Theory and Experiment, 2(2009)

[D] M. Duits, The Gaussian free field in an interlacing particle system with two jump rates; to appear in Comm. Pure and Appl. Math.; arXiv : math-ph/1105.4656

[K1] R. Kenyon, Dominos and the Gaussian free field; Ann. Probab. 29(2001), no. 3, 1128-1137.

[K2] R. Kenyon, Height Fluctuations in the Honeycomb Dimer Model; Comm. Math. Phys. 281(2008), no. 3, 675-709.

[Ku] J. Kuan, The Gaussian free field in interlacing particle systems; arXiv : math-ph/1109.4444

[P] L. Petrov, Asymptotics of Uniformly Random Lozenge Tilings of Polygons. Gaussian Free Field; arXiv : math-ph/1206.5123

[RV] B. Rider and B. Virág, The noise in the Circular Law and the Gaussian Free Field; Int. Math. Res. Not. (2007), article ID: rnm006

[V] M. Vuletić, The shifted Schur process and asymptotics of large random strict plane partitions; Int. Math. Res. Not. (2007), article ID: rnm043 